\title{
Pressure measurement in combusting and non-combusting gases using laser-induced grating spectroscopy
}

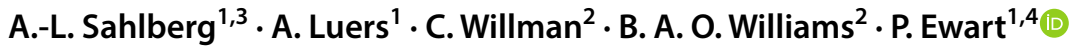

Received: 21 November 2018 / Accepted: 9 February 2019 / Published online: 15 February 2019

(c) The Author(s) 2019

\begin{abstract}
The measurement of pressure using laser-induced thermal grating spectroscopy, LITGS, with improved accuracy and precision is reported. Pressure values are derived from the record of the time-profile of LITGS signals by fitting of modelled signals to experimental data. The procedure is described for accurate modelling of the LIGS signals involving a sequence of calculation steps with appropriate weighting and calibration to determine the best-fit value of pressure-dependent parameters for averaged and single-shot measurements. Results are reported showing application of this model-fitting method to measurements of pressure in static cells using LITGS generated from NO in mixtures containing $\mathrm{N}_{2}$ at pressures in the range $0.5-5.0$ bar with accuracy of 1-3\% and single-shot precision of 4-7\%. Time-resolved measurements of pressure, using LITGS signals generated in toluene-seeded fuel vapour, during the compression and expansion strokes of a motored optically accessible engine are reported with pressure-dependent accuracy ranging from better than 10 to around $20 \%$ over the cycle and single-shot precision in the range $5-15 \%$ over the same range. Measurements in the engine under firing conditions were obtained over a limited range and slightly increased uncertainties associated with varying composition resulting from exhaust gas residuals. The method was found to be of limited utility for measurements in high temperature flames at around ambient pressures.
\end{abstract}

\section{List of symbols}

LITGS Laser-induced thermal grating scattering

MFM Model-fitting method

$P \quad$ Pressure

$T \quad$ Temperature

$\Lambda \quad$ Inter-fringe spacing of laser-induced grating

$c_{\mathrm{s}} \quad$ Local speed of sound in the gas

$f_{\text {osc }} \quad$ Oscillation frequency of LIGS signal

$\gamma \quad$ Ratio of specific heats of gas at constant pressure and volume

$m \quad$ Mean molecular mass of gas molecules

$k_{\mathrm{B}} \quad$ Boltzmann's constant

$\mu \quad$ Viscosity of the gas

P. Ewart

paul.ewart@physics.ox.ac.uk

1 Department of Physics, Oxford University, Parks Road, Oxford OX1 3PU, UK

2 Department of Engineering Science, Oxford University, Parks Road, Oxford OX1 3PJ, UK

3 Present Address: Department of Physics, Combustion Physics, Lund University, Box 118, 22100 Lund, Sweden

4 Present Address: Worcester College, Oxford OX1 2HB, UK $\tau_{\mathrm{o}} \quad$ Time delay off-set on LIGS signal relative to zero of time reference

$\tau_{\mathrm{g}} \quad$ The inter-fringe transit time given by $\Lambda / c_{\mathrm{s}}$

Re Reynolds number

$Q_{1} \quad$ The 'fast' quenching rate

$Q_{2} \quad$ The 'slow' quenching rate

$r \quad$ The branching ratio between the 'slow' and 'fast' quenching channels.

BBO Beta barium borate

TDC Top dead centre

CAD Crank angle degree

NIST National institute for standards and technology (USA)

\section{Introduction}

Pressure is an important parameter in a variety of technical situations involving combusting and non-combusting gases and in both static and dynamic conditions. The measurement of gas pressure is normally, and very effectively, achieved using transducers which are usually mounted in the wall of the containing vessel. In some situations, however, such as hostile or rapidly changing environments, or 
where a local pressure fluctuation occurs remote from the walls, such devices will not be able to record accurately any localised or dynamic pressure variations. In such cases a non-invasive or remote sensing capability can be advantageous. For example, dynamic pressure measurements are valuable in high-speed wind-tunnels and shock-tube studies of non-combusting flows. Localised pressure variations associated with pre-combustion chemistry are also important factors in auto-ignition and 'knock' effects in internal combustion engines. Few methods exist for making remote and non-invasive measurement of gas pressure that can provide time and space resolution. Pressure-broadening of spectral lines recorded using wavelength-modulated spectroscopy with $\mathrm{cw}$ lasers has been successfully employed for pressure measurements in gas flows. Spatial resolution was achieved by detection of fluorescence from a point and time-resolution by the rapid scan across the absorption lineshape [1, 2]. The technique was extended using tunable diode laser absorption spectroscopy, TDLAS, to give higher temporal resolution but a loss of spatial resolution [3]. These techniques require detailed knowledge of the line-broadening mechanisms determining the spectral lineshape. Spatially and temporally resolved measurements have been demonstrated using nonlinear optical processes such as CARS [4, 5]. More recently a technically simpler nonlinear technique of laser-induced grating scattering, LIGS, has been demonstrated for time- and space-resolved pressure measurement [6-9]. This paper reports on further developments of barometry using this technique.

The basic physics of LIGS, or laser-induced thermal acoustics, LITA, has been reviewed elsewhere and so is described only briefly here [10-12]. The process involves the interference of two degenerate frequency pump beams crossing at a small angle to produce a grating pattern. This grating is written into a gas medium by the mechanism of absorption and quenching or by electrostriction. Absorption and quenching processes lead to an exponentially decaying stationary temperature and density grating and the rapid density perturbation also induces two counterpropagating acoustic waves. The resulting standing acoustic wave modulates the scattering efficiency from the stationary thermal grating leading to a signal in the form of a decaying exponential with a super-imposed sinusoidal modulation at a frequency, $f_{\text {osc }}$, determined by the grating spacing $\Lambda$ and the local sound speed $c_{\mathrm{s}}$. In the case where the signal is dominated by absorption and quenching processes the technique is more precisely known as laser-induced thermal grating scattering, LITGS. In the absence of absorption, the electrostriction effect induces the standing acoustic wave alone, and this also provides a signal with an oscillating intensity, decaying by diffusion and viscous damping effects, but without the exponentially decaying background signal [11]. Measurement of the oscillation frequency of the LITGS signal, $f_{\text {osc }}$, yields a value for the speed of sound when the grating spacing, $\Lambda$, is known. The oscillatory behaviour of the signal may be characterised also by the time taken for the sound waves to cross the distance between the grating planes-the interfringe or grating transit time, $\tau_{\mathrm{g}}$. Measurement of $\tau_{\mathrm{g}}$ or $f_{\text {osc }}$, allows the temperature to be derived from the speed of sound using an appropriate equation of state. The decay rate of the signal is determined, inter alia, by the pressuredependent diffusion and viscous damping rates. Thus, in principle, the pressure may be derived from measurement of the signal decay rate.

LITGS signals from thermal gratings have been used to measure temperature and pressure at a point in a highpressure flame although only in the high-pressure regimes between 10 and 40 bar. The accuracy in this work was limited to around $7 \%$ at the highest pressures and $22 \%$ at $20 \mathrm{bar}$ with single-shot precision in the region of $10 \%$ [6]. In cellbased studies the accuracy was improved by a more detailed treatment of relaxation and quenching processes achieving a single-shot precision of $1.4 \%$ and accuracy, limited by uncertainty in the values of gas kinetic parameters, of 5.7\% over the whole pressure range of 3-40 bar [8]. The method has also been extended to give measurements of temperature and pressure along a 1-D line [9]. Since the duration of the LITGS signal decreases with decreasing pressure, as a result of faster decay of the grating by molecular diffusion, measurements using the technique are less accurate and precise in lower pressure environments such as, for example, highspeed flows. Hart et al. measured pressure in a supersonic (low pressure) air flow using the pressure-dependent decay of the two counter-propagating acoustic wave packets, or gratings, launched at the speed of sound $c_{\mathrm{s}}$, upstream and downstream of the flow. The two wave packets arrived at a displaced probe beam at different times since, in the laboratory frame, their speed was increased or decreased by the flow velocity vector. The different signal strengths gave a measure of the pressure-dependent decay of the acoustic wave from which the pressure was derived with an uncertainty of around 4\% [13].

The present work extends the previous pressure measurements to a range of lower pressures in both cell-based studies and in a gasoline direct injection (GDI) internal combustion engine. In general, the relationship between the signal decay and pressure is complex and this work presents a refined approach to deriving the pressure from measured LITGS signals. This method, the model-fitting method, MFM, relies on fitting of a theoretically modelled signal to the experimental data using pressure-dependent parameters to obtain a best-fit value for the pressure. The application of the method to cellbased measurements in $\mathrm{NO} / \mathrm{N}_{2}$ mixtures and to in-cylinder measurements in an optically accessible GDI engine using toluene absorption in gasoline fuel/air mixtures is reported. 
In addition the application of the method for pressure measurement in flames at around ambient pressures is briefly discussed.

\section{Derivation of pressure from LITGS signals}

In general the derivation of pressure from LITGS signals presents significant theoretical and data analysis challenges. In the first application of the method, using excitation of thermal gratings in the $\mathrm{OH}$ radical in a high-pressure methane/air flame, a simple approach was used to calculate model signals based on the solution to the linearized hydrodynamic equations governing the evolution of the induced grating [6, $14,15]$. At lower pressures the uncertainty increased owing to inadequacies of the model regarding the quenching times that are comparable to the timescale of the grating evolution and decay. Calculation of accurate model signals based on the equations describing the dynamics of the induced grating evolution is a difficult computational problem even when certain simplifying assumptions are made, e.g., the time to establish the grating is much shorter than the grating transit time, $\tau_{\mathrm{g}}$, and the Reynolds number is large, $R e \gg 1$. The source function describing the grating evolution must also include effects having timescales that are similar or greater than $\tau_{\mathrm{g}}$, such as quenching times and the duration of the pump pulses (typically $~ 10 \mathrm{~ns}$ ). A numerical solution involves a computationally stiff problem especially when fitting of the model signal to experimental data. This issue was addressed by Stevens and Ewart using a Fourier domain approach that could more readily incorporate quenching processes occurring on different time scales and other experimentally determined parameters such as laser pulse duration, etc. [8]. This approach achieved reasonable results in pressure determination from LITGS signals in $\mathrm{NO}_{2} / \mathrm{N}_{2}$ mixtures in the range of 3-40 bar.

\subsection{Derivation of pressure by fit of model to experimental signals: model-fitting method, MFM}

The present work is also based on computation of a model LITGS signal and finding the best fit to experimental data-the model-fitting method, MFM. As in previous work, the model used to calculate a "theoretical" LITGS signal is based on the general approach of Paul et al. using a set of linearized hydrodynamic equations describing the evolution, i.e. growth and decay, of the induced grating and acoustic waves [8, 14]. The signal is determined by geometrical parameters determining the dimensions of the grating, the medium properties, including thermodynamic and gas dynamic parameters, which determine the evolution of the grating and the speed of sound. The grating transit time, $\tau_{\mathrm{g}}$ and the Reynolds number, $R e$, incorporate several of these factors. The energy transfer to the bulk gas medium following molecular absorption proceeds, in general, by several quenching mechanisms and internal energy conversion processes.

It is possible, in principle, to model the quenching process involving multiple decay channels [8, 14]. This has been done in some special cases where the decay routes can be identified with known energy level structures in the molecule [16]. In other cases it is possible to model complex decay cascades by assuming only two channels characterised by a fast and a slow decay rate. The slow channel represents by a single process, with a single decay rate, a chain of sequential decay processes [17]. In a similar manner, the model used here approximates these relaxation mechanisms by assuming two quenching channels with different rates, $Q_{1}$ and $Q_{2}$. This approach was found previously to provide accurate values of temperature and pressure by fitting to LIGS signals in $\mathrm{NO}_{2}$ in cell-based studies [8]. The branching ratio, $r$, describing the relative contribution of each decay route, is specified here by the proportion decaying by the 'slow' channel at rate $Q_{2}$. These factors lead to five parameters that may be used to characterise the LITGS signal;

- $\tau_{\mathrm{g}}$, the inter-fringe transit time given by $\Lambda / c_{\mathrm{s}}$

- $R e$, the Reynolds number

- $Q_{1}$, the 'fast' quenching rate

- $Q_{2}$, the 'slow' quenching rate

- $r$, the branching ratio between the 'slow' and 'fast' quenching channels.

In practice an experimental LITGS signal is recorded relative to some time-zero reference, usually associated with the triggering of the oscilloscope or data recording device. Therefore, an additional parameter specifying the "time-offset", $\tau_{\mathrm{o}}$, needs to be adjusted to align the model signal to the experimental data for fitting purposes. Thus six parameters are required to model the signal and it is, in principle, possible to determine values giving a "best fit" to the data. In particular, the determination of the value of the Reynolds number provides a parameter from which the pressure, $P$, may be derived using the relation,

$P=\frac{\mu k_{\mathrm{B}} T R e}{c_{\mathrm{s}} \Lambda m}$,

where $\mu$ is the viscosity of the gas, $k_{\mathrm{B}}$ is the Boltzmann constant, $T$ is the temperature and $m$ is the mean molecular mass of the gas. This equation is derived simply from the equation of state for an ideal gas and the definition of the Reynolds number using the sound speed, $c_{\mathrm{s}}$, as the characteristic velocity and the grating spacing $\Lambda$ as the characteristic 
length. Derivation of the pressure by use of this relation is a reasonable approach since the ideal gas equation remains valid throughout the range of pressures encountered in the present work. Applications involving diesel engine conditions of very high pressures may require modification as a result of changes to the gas dynamic parameters. Such modifications have been employed in previous work using LIGS at elevated pressures in a compression machine to simulate diesel engine conditions [18].

It will be apparent immediately that a "fitting" in a sixdimensional parameter space comprised of $\tau_{\mathrm{o}}, \tau_{\mathrm{g}}, \operatorname{Re}, Q_{1}, Q_{2}$, and $r$ is unlikely to produce a single, well-defined value for each of the parameters. The problem is, however, not totally intractable since the physics of the situation will constrain values within limits that can be reasonably estimated and it will usually be possible to give a sufficiently accurate estimation to provide starting values for an iterative fitting procedure. Nonetheless, having several parameters that affect the signal decay in a similar manner will almost certainly provide local minima that will not correspond to accurate values of the parameters in question. This is particularly true in the present case, where the parameters are not all orthogonal. In particular, the decay rate will be affected by both $R e$ and $Q_{2}$ and gas dynamic parameters are affected by both temperature and pressure. This challenge is addressed by finding first those parameters that are independent, or approximately so, and second those that are, at least within an acceptable range, independent of temperature and pressure. Calibration procedures are then used to account for variation in the other parameters over the range of experimental conditions. In addition, by considering the sensitivity of the signal to the various parameters, it is possible to minimise errors by suitable weighting of the fitting procedure to parts of the signal that are less sensitive to errors in some of the parameters. For example, the fast quenching rate, $Q_{1}$, directly affects the build-up time of the grating and thus affects more significantly the first part of the signal. Therefore, by weighting the fit to the later parts of the signal, which are still sensitive to pressure, the effects of errors in $Q_{1}$ are minimised. A four-stage fitting procedure was adopted to take advantage of the independence of several of the parameters.

The first step, however, inevitably requires making an educated guess for the values of each of the parameters. Fortunately, it is possible to make a reasonable estimation of the two time parameters, $\tau_{\mathrm{o}}$ and $\tau_{\mathrm{g}}$, from the observed signals. The "time-offset", $\tau_{\mathrm{o}}$ can be determined with reasonable accuracy and precision by measurement of the time delay between the arrival of the pump pulse and the start of the LITGS signal itself by recording both on a 2-channel oscilloscope. The value of $\tau_{\mathrm{g}}$ is readily estimated directly from the signal or from the inverse of the oscillation frequency, $f_{\text {osc }}$, found by a Fourier transform of the signal.
The second step involves assuming some values for $Q_{1}$, $Q_{2}$, and $r$ to find a value for $R e$. Here, again, we may make a physically reasonable assumption that the "fast" rate, $Q_{1}$, will be less than the inter-fringe transit time and, based on a realistic collision rate at the range of pressure expected, a value of 1-10 ns is assumed for the "fast" quench time, i.e. $Q_{1}, \sim 10^{9}-10^{8} \mathrm{~s}^{-1}$. The time scale associated with the "slow" quench rate is then assumed to be in the range $\sim 50-300 \mathrm{~ns}$, where the lower bound is set to be approximately ten times the fast quench time and the upper bound is based on the duration of the typical LITGS signal observed. Thus $Q_{2}$ is set to the range $2 \times 10^{7}-3 \times 10^{6} \mathrm{~s}^{-1}$. The initial value of $r$ is more arbitrary but must allow for most of the energy to be transferred by quenching fast enough to generate an observable oscillatory signal. Typical "starting" values of $r$, defined by the proportion of the branching to the slow channel, will, therefore, be in the range 0.05-0.3. Using the values estimated for $\tau_{\mathrm{o}}, \tau_{\mathrm{g}}$, with fixed "starting" values for $Q_{1}, Q_{2}$, and $r$, with an initial guess at $R e$, the fit may be optimised to find a "best fit" value for $R e$.

The third step consists of fixing the values of $\tau_{\mathrm{o}}, \tau_{\mathrm{g}}$ and $R e$ and adjusting the values of $Q_{1}, Q_{2}$, and $r$ to find the "best fit". The fourth, and final, step is to allow all parameters to vary, starting with the optimised initial values obtained in steps $1-3$, in a nonlinear least-squares fitting to find an optimum set of values for all the parameters.

The description of the fitting procedure so far has ignored the fact that different parameters can affect the signal in the same way. For example, the signal decay rate is affected by both the value of $R e$ and the quenching rates, with $Q_{2}$ having a particularly strong influence. Similarly, the effects of $Q_{1}$ and $r$ can also not be totally ignored. To address this problem, a calibration procedure is used to constrain the values of $Q_{2}$ and $r$ and so the fitting procedure at step 3 will be allowed to focus on optimising $Q_{1}$. Residual uncertainties in $Q_{1}$ will subsequently be addressed by a weighting procedure. The "fixing" of $Q_{2}$ and $r$ is, of course, a simplification but it turns out to be valid over a large range of temperature and pressure. This was verified by fitting to the signals under the calibration conditions over the range from 0.5 to 5.0 bar as shown in Fig. 2 in addition to two conditions in the engine at low and high pressure and temperature.

Calibration was achieved by recording a LITGS signal at a chosen "calibration" point close to atmospheric pressure and room temperature. The values of $Q_{2}$ and $r$ were selected after running an exhaustive search across both parameters to obtain the pair of values yielding a value of derived pressure closest to that recorded on a calibrated pressure gauge. Some residual uncertainty remained since several combinations of $Q_{2}$ and $r$ could lead to equally good fits. However, it was found that variation of the decay time for rate $Q_{2}$ in a range of $\pm 10 \mathrm{~ns}$ led to an error of only $\pm \sim 4 \%$ in the derived pressure. 
The sensitivity of the fitting procedure to the value of $Q_{1}$ was reduced by the following weighting process. The fast quenching rate has its strongest effect on the initial buildup of the signal as the induced grating develops. Thus, by weighting the fitting process to the later stages of the signal the effects of uncertainty in $Q_{1}$ are minimised. A, somewhat arbitrary, objective criterion was set by which the area under the signal curve was calculated to determine the time at which $50 \%$ of the signal has been produced. The fitting at stage 4 was then constrained to find the best fit to only that part of the signal that came later than this $50 \%$ point. In practice it was found that cutting off the first $30 \%$ rather than $50 \%$ produced a very similar result. Changing the fraction cut-off from 0 to $60 \%$ led to a variation in the derived pressure of only 6-7\%. Although difficult to quantify, this degree of uncertainty, in the absolute value of the derived pressure, was deemed acceptable to reduce the potentially more serious errors introduced by uncertainty in the value of $Q_{1}$.

The procedure developed for fitting a modelled LIGS signal to experimentally acquired signals described above was implemented using MATLAB ${ }^{\circledR}$.

\subsection{Temperature effects}

Having derived a "best fit" value for $R e$, the pressuredependent parameter, account must be taken of the temperature, $T$, and the temperature dependence of $\mu$, the viscosity, and $\gamma$, the ratio of specific heats at constant pressure and volume, for the gas mixture. The temperature is first estimated from the measured value of $f_{\text {osc }}$, using an approximate value for $\gamma$ :

$T=f_{\mathrm{osc}}^{2} \Lambda^{2} \frac{m}{\gamma k_{\mathrm{B}}}$.

A more precise value of $\gamma$ is then obtained from the NIST data base, Reference Fluid Thermodynamic and Transport Properties-REFPROP, before iterating to a consistent solution for $\gamma$ and $T$ [19]. The viscosity $\mu$ is then also obtained from the database, allowing for further iteration to accommodate any slight pressure dependence in the database. We note that, following Eq. 1, any error in the tabulated value of $\mu$ from REFPROP will directly affect pressure. Any error in the REFPROP value of $\gamma$ will also affect pressure, but this comes as a result of determining $T$ incorrectly, in turn causing the wrong value of $\mu$ to be selected. This is not possible to directly quantify since the exact form of the dependence of $\mu(T)$ is not known analytically. In the limit of elastic scattering of spheres, $\mu(T) \propto T^{1 / 2}$, which indicates that inaccuracy in tabulated values of $\gamma$ is less important compared to $\mu$.

\subsection{Composition effects}

To choose the correct value of $\gamma$ and also of the mean molecular mass, $m$, however, knowledge of the gas composition is required. For cell-based measurements, where the gas composition is known, the appropriate values can be determined with good accuracy. For measurements in engines, on the other hand, it may be necessary to make some approximations based on the best estimate of the gas composition. The effects of uncertainties in gas composition have been discussed in detail previously for temperature measurements using LITGS in a GDI-spark ignition engine [20]. This previous work showed that early injection of the fuel in a motored DI engine produced a sufficiently homogeneous mixture such that local variations in composition were insignificant. When the engine was fired, the composition was changed by the presence of exhaust gas residuals. However, this work showed also that these did not appreciably affect the value of $\gamma / m$ for the mixture. The impact of composition on quenching rates will be considered in Sect. 3.2. When measurements are based solely on thermal grating scattering, resulting from absorption in a fuel component such as toluene, derivation of both temperature and pressure is possible only during the intake/compression stroke up to the point where the absorber is consumed by combustion.

\section{Experimental apparatus and procedure}

Experiments to test the procedure for deriving pressure from LITGS signals were performed primarily in two situations-in gas cells at constant temperature and variable pressure and in a motored optical engine, i.e. a spark ignition engine fitted with windows in the upper section of the combustion chamber. The engine provided an environment in which the temperature varied during a polytropic process and measurements could be made at different crank angle degrees (CAD) during the compression and expansion stroke. Some additional measurements were made in a methane/oxygen flame at slightly elevated pressures.

\subsection{Cell-based measurements of pressure}

In the cell-based experiments (see Fig. 1) the signals were generated in $\mathrm{NO}$ at a partial pressure of $3.5 \mathrm{mbar}$ in $\mathrm{N}_{2}$ added at pressures in the range $0.5-5.0$ bar. The gas mixture was contained in a stainless steel cell fitted with fused silica windows to transmit the input pump and probe beams and the output signal beam. To create a thermal grating, pump laser pulses at $226 \mathrm{~nm}$ were used to excite absorption transitions in the $(0,0) \gamma$-bands of NO. The laser system used consisted of a Q-switched Nd:YAG laser (Continuum Powerlite 8000) operating at $10 \mathrm{~Hz}$ and emitting both the second and third 


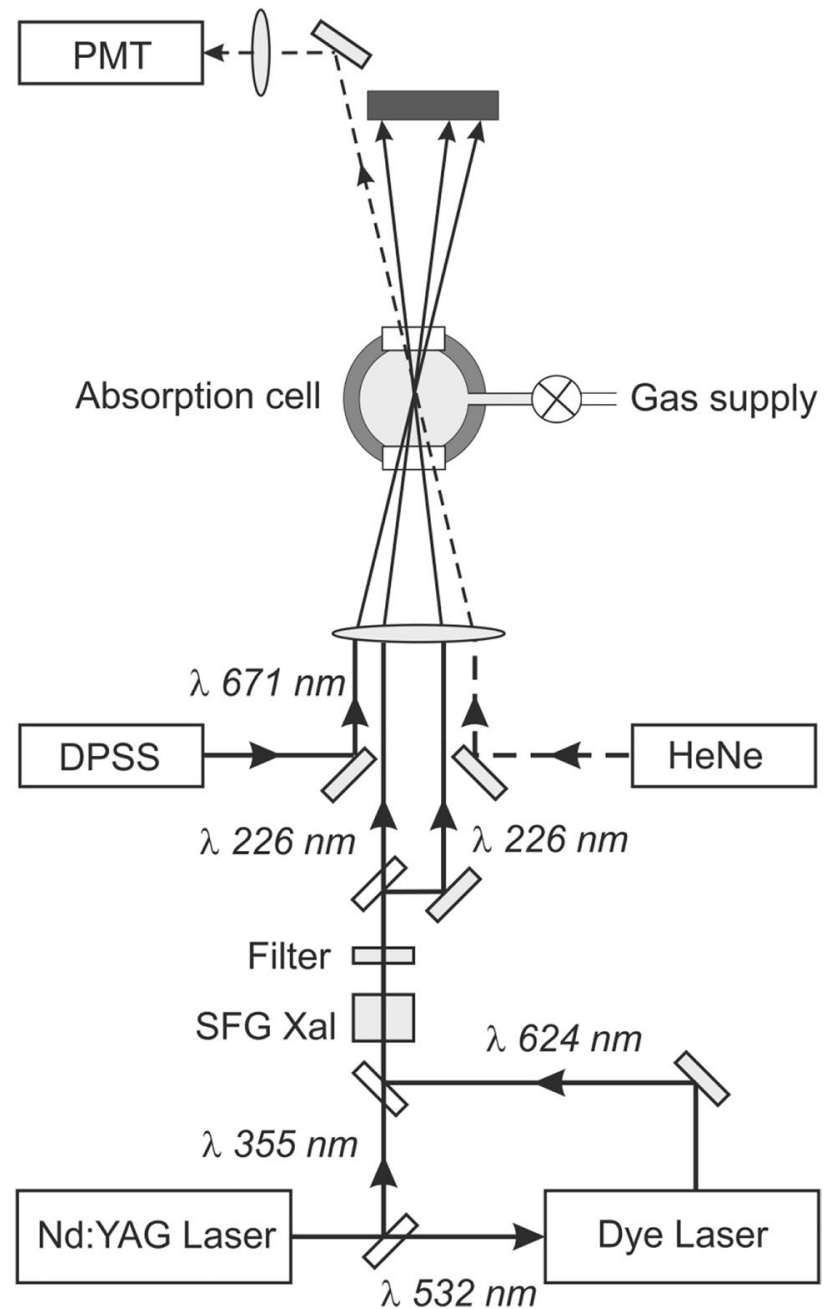

Fig. 1 Schematic representation of the experimental layout for cellbased LITGS measurements in NO: $\mathrm{N}_{2}$ mixtures. PMT photomultiplier tube (for detection of signal), DPSS diode-pumped solid state laser, providing probe beam at $671 \mathrm{~nm}, \mathrm{HeNe}$ helium-neon laser used for alignment of signal beam to detector, $S F G \mathrm{Xal}$ sum frequency generation crystal mixing beams at $355 \mathrm{~nm}$ and $624 \mathrm{~nm}$ to produce pump beams at $226 \mathrm{~nm}$. The Nd:YAG laser emits both third and second harmonic beams at $355 \mathrm{~nm}$ and $532 \mathrm{~nm}$, respectively, that are separated by a dichroic beam splitter, with the $\lambda 532 \mathrm{~nm}$ beam used to pump the dye laser

harmonics at $532 \mathrm{~nm}$ and $355 \mathrm{~nm}$, respectively. The second harmonic was used to pump a modeless dye laser whose output was spectrally narrowed to a linewidth of approximately $0.3 \mathrm{~cm}^{-1}$ [21]. Radiation at $226 \mathrm{~nm}$ was generated by sum frequency mixing, in a BBO crystal, the output of the dye laser, tuned to a wavelength of $624 \mathrm{~nm}$, with the third harmonic of the pump laser at $355 \mathrm{~nm}$. The precise wavelength of $226.12 \mathrm{~nm}$ was inferred from measurements of the wavelength of the Nd:YAG harmonic outputs and of the dye laser using a pulsed wavemeter (Burleigh WA-4500). The resulting output consisted of pulses of $5 \mathrm{~ns}$ duration and

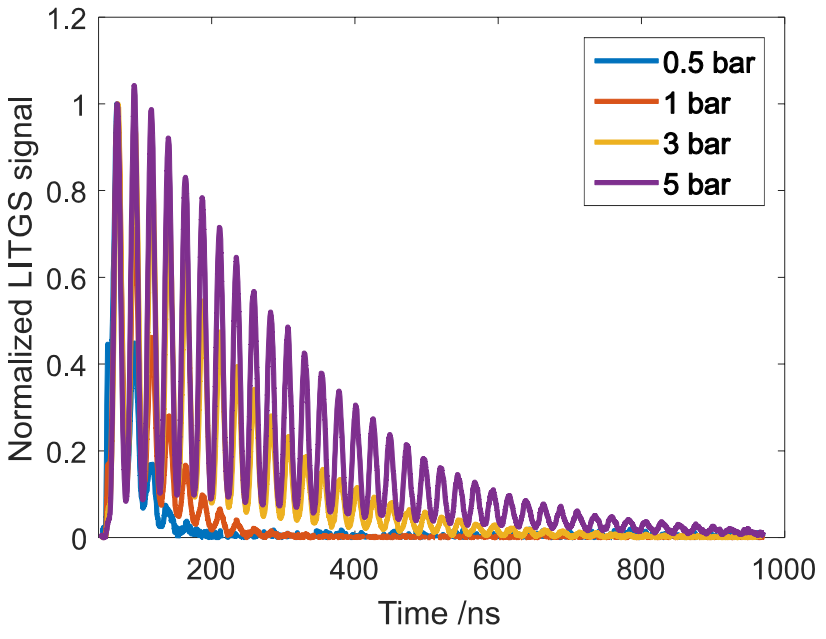

Fig. 2 LITGS signals from $\mathrm{NO}$ at various pressures of $\mathrm{N}_{2}$ (see text for details), 0.5 bar-blue, 1 bar-red, 3 bar-orange, 5 bar-purple. The signals are averages over 100 single shots at each pressure

$1 \mathrm{~mJ}$ energy which was sufficient to generate LITGS signals with adequate signal-to-noise ratio.

The temperature in the cell was monitored by a calibrated thermocouple in contact with the cell wall. The temperature was measured using the LITGS signals averaged over 100 successive single shots of the laser system. The uncertainty in the measurement was indicated by the standard deviation of $0.7 \mathrm{~K}$ at a room temperature of $295 \mathrm{~K}$, i.e. a single-shot precision of $0.2 \%$.

A set of LITGS signals, averaged over 100 shots per set, is shown in Fig. 2 for pressures ranging from 0.5 to 5 bar of $\mathrm{N}_{2}$. The increase in decay time of the signal with increasing pressure measured by a pressure gauge, is clearly evident in this data.

To derive the pressure from the LITGS data, as explained above, it was necessary to decide upon the appropriate values of the Reynolds number, quenching rates, $Q_{1}$ and $Q_{2}$ and the branching ratio, $r$. A sensitivity study showed that the optimum $Q_{2}$ varied little with temperature and pressure over the range studied. The decay rate of the signal, in this range, is insensitive to $Q_{1}$ as this rate affects mostly the initial build-up of the induced grating, its effects becoming less significant at longer times after the initial excitation. A value of the decay time for rate $Q_{2}=60 \mathrm{~ns}$ with $r$ of 0.05 provided a good fit at the calibration point at 1 bar total pressure at room temperature. The data and fit are shown in Fig. 3. Variation of the decay time for rate $Q_{2}$ by $\pm 10 \mathrm{~ns}$ introduced an error of only \pm 0.02 bar.

Pressure values were then derived from the acquired LITGS data using the MFM and compared to the values obtained from the pressure gauge. The results are shown in Fig. $4 \mathrm{a}$ and show reasonable agreement with values derived from LITGS, averaged over 100 single shots. The accuracy 


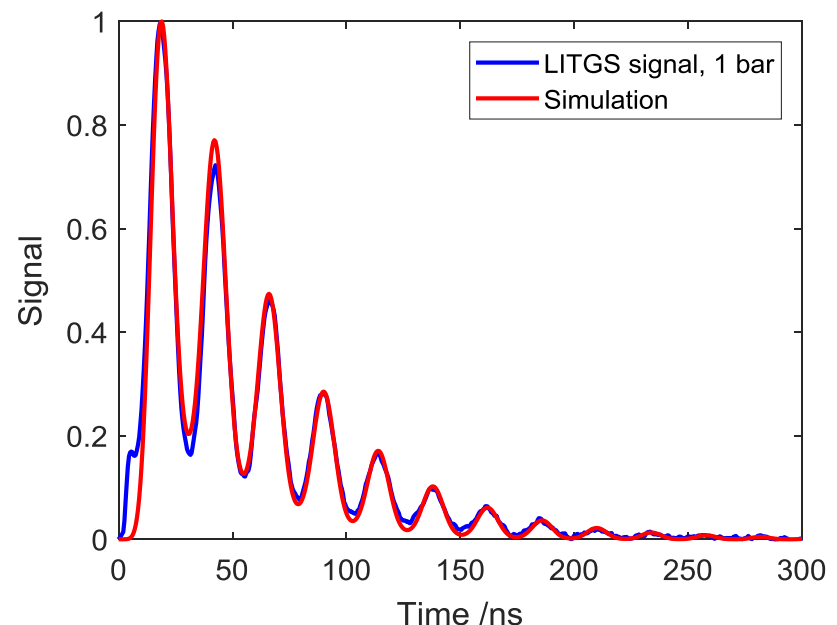

Fig. 3 LITGS signal, averaged over 100 single shots, (solid blue line) and model fit (dashed red line) using the 'best fit' value for 'slow' quench rate, $Q_{2}$, of $60 \mathrm{~ns}$ and branching ratio $r$ of 0.05

of the derived pressure values, relative to the value obtained using the pressure gauge, is between $1-3 \%$, or $20-79$ mbar, except for the first data point around 0.5 bar where the deviation is $6 \%$. The single-shot precision, based on the standard deviation of the individual measurements, varies between 4 and $7 \%$, i.e. $0.1-0.2$ bar. The lowest data point at 0.5 bar, however, is again an exception where the uncertainty is $20 \%$. The reduced precision of the measurements at the lowest pressure reflects the shorter duration of the LITGS signals under these conditions. These results represent a significant improvement in pressure measurement accuracy and precision over previous work where similar experimental

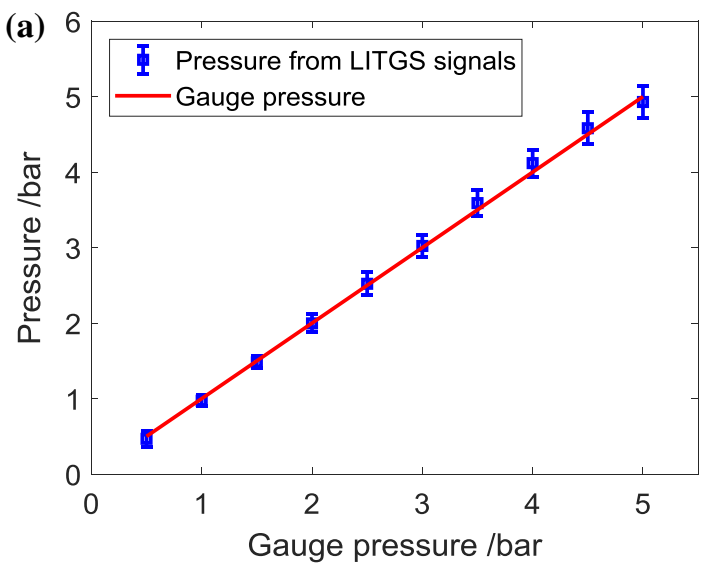

Fig. 4 Comparison of pressure values derived using LITGS, averaged over 100 single shots and analysis by the MFM, with those from a pressure gauge (see text for details), a data points (blue) are the values derived from the LITGS measurements and the solid line (red) is the line indicating equality with the measurement by the pressure gauge. Deviation of the data points from this line, therefore, gives an uncertainties were achieved only at high pressures in the $10-40$ bar range. The gradual increase in error with increasing pressure suggests that the assumptions made regarding the constancy of quench rates and branching ratio break down with increasing difference in pressure from the calibration point.

\subsection{Engine-based measurements of pressure}

LITGS measurements were performed in a single-cylinder optical engine, based on a Jaguar Land Rover AJ133 design, which was fitted with four fused silica windows $(16 \mathrm{~mm}$ clear aperture) arranged at each quadrant of a metal annulus sandwiched between the barrel and the cylinder head. The optical arrangement of the laser beams is shown in Fig. 5 . To obtain data around the full cycle, including the point of maximum compression, a custom piston was manufactured with a rectangular slot machined to allow the pump and probe beams to be transmitted when the piston was at top dead centre (TDC) [22]. The crown of this "slotted piston" was shaped to compensate for the "missing" volume to maintain the same compression ratio. Fuel consisting of $30 \%$ toluene and $70 \%$ iso-octane was mixed with air in a preheated plenum at an intake pressure of -0.479 bar (gauge) and maintaining an equivalence ratio of 1 . The toluene concentration was then typically around $0.5 \%$ and maintaining the toluene absorber concentration at this low level avoided any significant perturbation to the temperature in the measurement volume as a result of energy absorption from the pump beams. Previous studies have shown that for toluene concentrations below $0.5 \%$ the perturbation to the local temperature is less than $1 \%$ and consequently, under ideal gas



indication of the accuracy. b Variation in fractional error in measurement of pressure by LITGS. The horizontal lines, green and red, indicate the fractional error of $10 \%$ and $20 \%$, respectively. The error bars indicate the standard deviation of the single-shot measurements and give a measure of the single-shot precision 
Fig. 5 a Schematic of arrangement of system of lasers and optics to produce pump and probe beams for generating LITGS signals. $L$ lens, $M$ mirror, $B S$ beam splitter, $P$ prism, $C P$ compensator plate. See text for details. b Optical arrangement of pump and probe beams for LITGS measurements in the optical engine (shown in section to indicate opposed windows in the cylinder head for optical access)

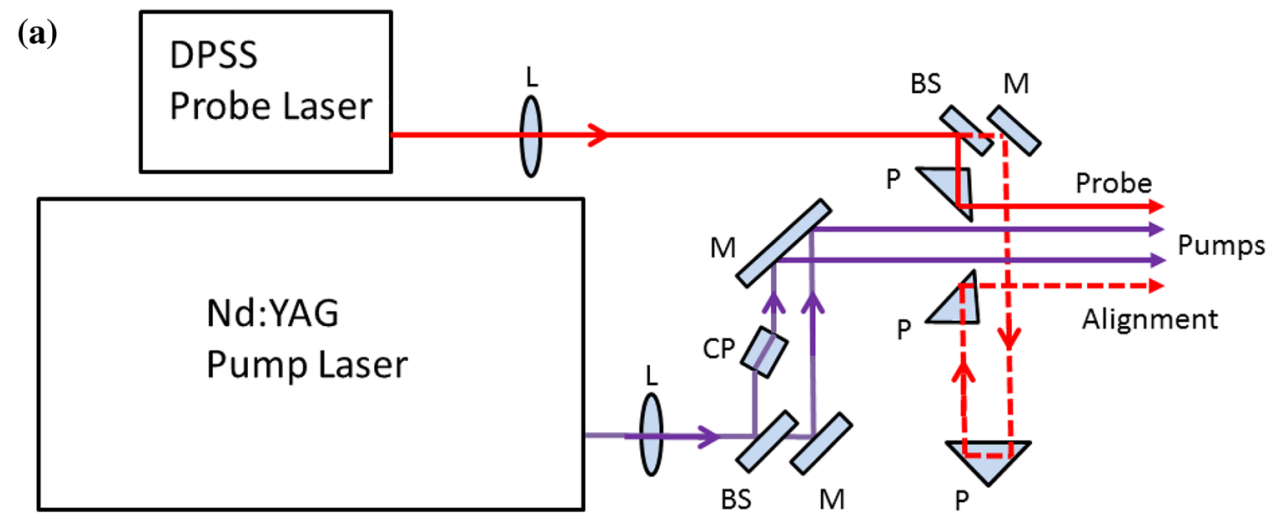

(b)

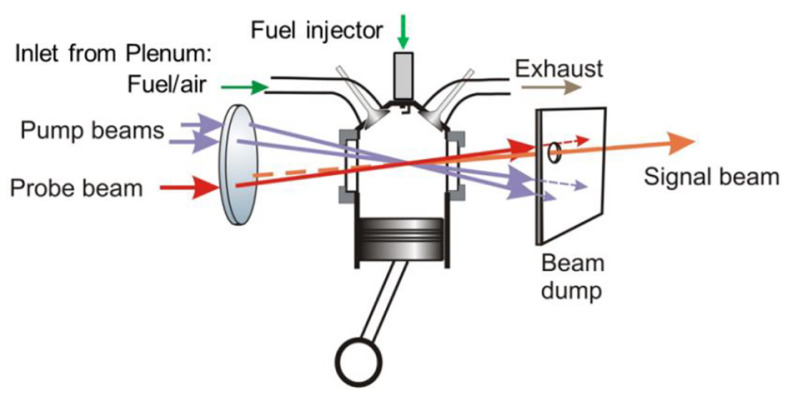

conditions, any perturbation of the local pressure will also be less than $1 \%$ [23].

The optical system used to generate the LITGS signals consisted of a portable device containing a Nd:YAG pump laser (Continuum Minilite II) and a cw probe laser (CNI MLL-H-671). The fourth harmonic of the Nd:YAG laser provided about $4 \mathrm{~mJ}$ at $266 \mathrm{~nm}$ in a $5 \mathrm{~ns}$ pulse at $10 \mathrm{~Hz}$. The laser could be triggered in synchronism with the engine to make measurements at a particular time during the engine cycle (specified in crank angle degrees, $\mathrm{CAD}$ ). The cw probe laser emitted $500 \mathrm{~mW}$ of light at $671 \mathrm{~nm}$, a fraction of which was used to provide a guide beam along the direction of the LITGS signal to facilitate alignment of the photomultiplier detector. The whole system consisting of the pump and probe lasers, beam splitting and directing optics was mounted on a compact base close to the engine so that the measurement volume could be conveniently positioned in the centre of the engine cylinder about $33 \mathrm{~mm}$ below the top of the pent-roof cylinder head. The spatial extent of the measurement volume was determined primarily by the diameter and narrow crossing angle of the pump beams, $2.5^{\circ}$, to be approximately $1 \mathrm{~mm}$ in diameter and $10 \mathrm{~mm}$ in length [24].

Representative examples of LITGS signals from the optical engine under motored conditions (no combustion) are shown in Fig. 6. These figures show the LITGS signals, averaged over 50 single shots, recorded at four points in the compression and exhaust stroke labelled by the crank angle degree (CAD) relative to top dead centre (TDC). The bestfit model signals, for each case, calculated using the MFM described above, are also shown. The effect of weighting the fit to the later part of the signals is apparent in the observed discrepancy between the modulation depth of the signal and simulation during the first few oscillations of the signal. As noted above this weighting aimed to minimise the dependence on the less well-known parameters that affect more strongly the initial phases of the signal. This discrepancy does not significantly affect the accuracy of the derived pressure which depends more on the fit to the later stages of the decaying signal. The gas composition was assumed to be $76.66 \% \mathrm{~N}_{2}, 20.66 \% \mathrm{O}_{2}, 1.65 \%$ iso-octane/toluene fuel and $1.03 \% \mathrm{Ar}$, which gives a mean molar mass of $\sim 30.4 \mathrm{~g} / \mathrm{mol}$. The values for the ratio of specific heats, $\gamma$, were estimated for the range of conditions using data from the NIST tables with the temperature-dependent values chosen in accord with the temperature derived from the measured oscillation frequency in an iterative procedure [19].

Derivation of the pressure was based on the determination of the Reynolds number according to Eq. (1) and the optimization of the fit-values for the other parameters. The value of $Q_{1}$ was found to be not critical so a value of $5 \mathrm{~ns}$ was chosen for the decay time since values between 2 and $8 \mathrm{~ns}$ provided similarly good fits together with a range of values in the other parameters. The most critical parameters were found to be the value of $Q_{2}$ and $r$, and an initial value was determined by adjustment to give the best fit at a calibration point in 

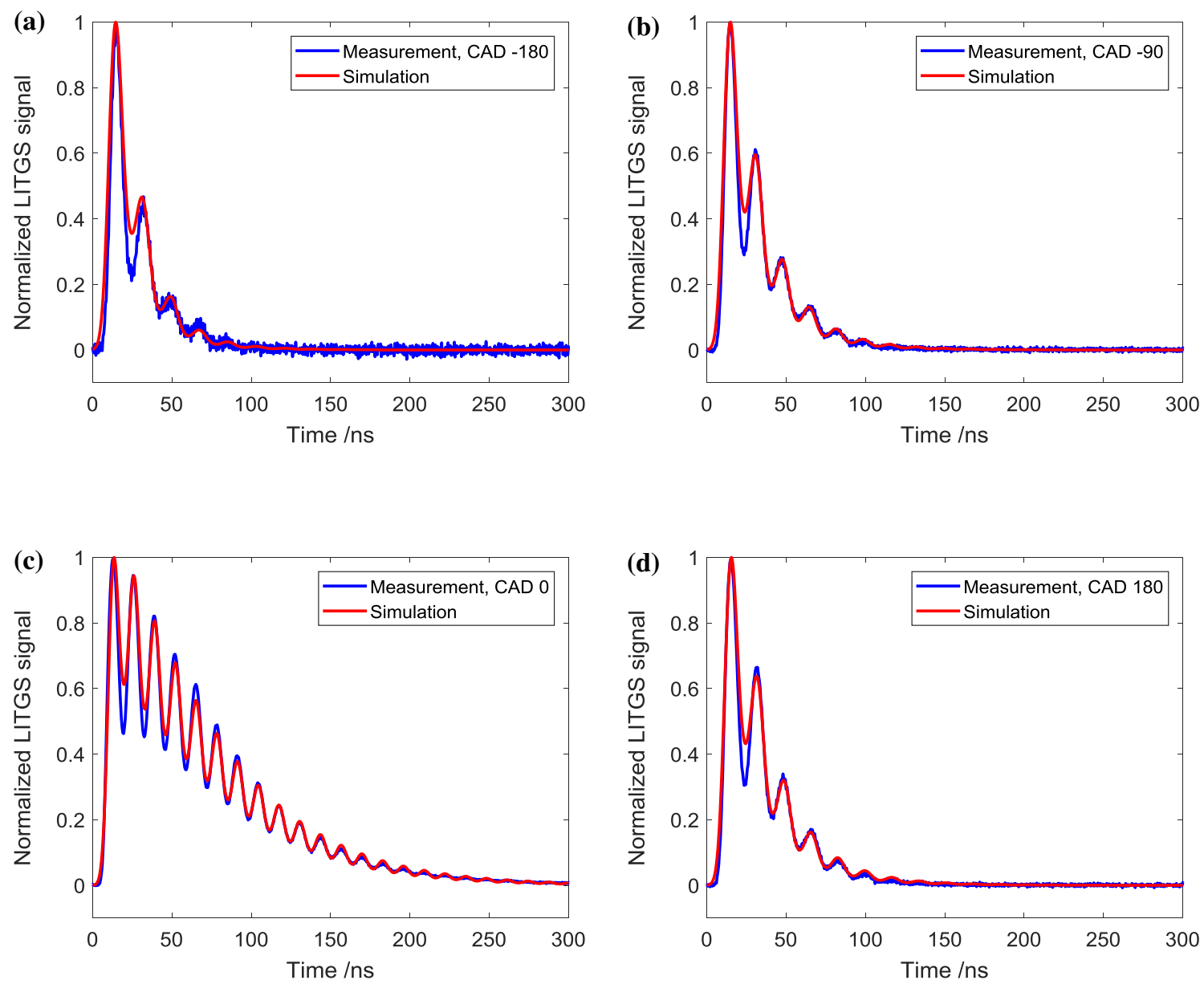

Fig. 6 LITGS signals from optical engine under motored conditions, averages of 50 single shots (blue lines) obtained at CAD a 180, b 90, c 0 and d 180 with best-fit model simulations (red lines). Note that

the fitting is weighted to the later part of the signal, hence the lessthan-optimum fit to the first few oscillation cycles in the signal (see text for details)

comparison with a value from the pressure transducer. Using a calibration point at which the transducer registered around $1 \mathrm{bar}$, a value of $60 \mathrm{~ns}$ for the decay time for rate $Q_{2}$ with $r=0.1$, gave optimal fitting over the range of CAD (and incylinder pressures $\sim 1-6$ bar) as shown in Fig. 7a. An indication of the sensitivity of the derived pressure to the value of $Q_{2}$ may be gauged by the fact that a change in the assumed value of the decay time for rate $Q_{2}$ of \pm 10 ns results in a change of only $5 \%$ (or $\sim 0.2$ bar) in the derived pressure at TDC. These results suggest that the analysis using the MFM of the LITGS data provides good accuracy over most of the pressure range encountered over the engine cycle.

The data shows that the accuracy and precision of the pressure values derived from the LITGS measurements relative to the pressure transducer values varies during the cycle, i.e. with changing pressure (and temperature) as shown in Fig. 7. Broadly speaking, two regimes can be identified for CAD values corresponding to pressures below and above 1 bar, with a maximum pressure of around 6.5 bar. At

pressures above 1 bar the accuracy, defined by the deviation from the pressure transducer value, is better than $\pm 10 \%$, $\left(0.4 \mathrm{bar}\right.$ at $\left.\mathrm{TDC}, \mathrm{CAD}=0^{\circ}\right)$. Within this range the singleshot precision, given by the standard deviation over 100 data points, lies between \pm 3.5 and $10 \%$. At pressures below 1 bar the relative uncertainty increases with an accuracy of typically $\pm 20 \%$ or 0.1 bar and a precision of $\pm 15-20 \%$. The variation in the relative error with pressure may be due to the difficulty of accurately fitting to the shorter duration signals observed at lower pressure that provide fewer data points.

Measurements in the engine under firing conditions encounter additional complications owing to added uncertainties in the gas composition and temperature variations. Furthermore, combustion consumes the toluene tracer along with the fuel and so measurements are possible only up to around $\mathrm{CAD}-10^{\circ}$.

The gas composition was assumed (estimating $15 \%$ of the in-cylinder gases to be combustion products from the previous cycle) to be $76 \% \mathrm{~N}_{2}, 17.4 \% \mathrm{O}_{2}, 1.39 \%$ iso-octane, 


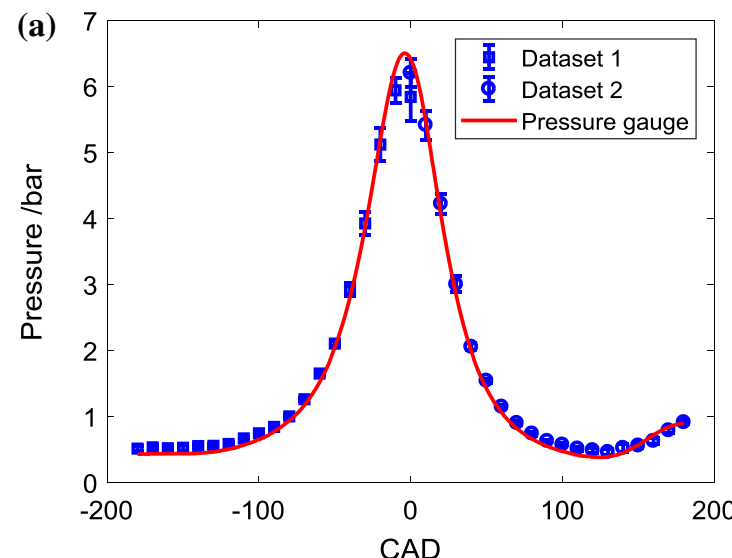

Fig. 7 LITGS measurements of in-cylinder pressure in motored engine using the MFM using a value of $Q_{2}$ of 60 ns (a). Datasets 1 and 2 were obtained during compression and expansion strokes, respectively. The pressure recorded by the pressure transducer is also

$1.97 \% \mathrm{CO}_{2}, 1.02 \% \mathrm{Ar}$ and $2.21 \% \mathrm{H}_{2} \mathrm{O}$, which gives a mean molar mass of $\sim 30.16 \mathrm{~g} / \mathrm{mol}$. The values for specific heat ratio, $\gamma$, and viscosity, $\mu$, were again estimated from NIST data using temperature values derived from the LITGS signals [19]. Under these conditions an optimum value for $Q_{2}$ of $40 \mathrm{~ns}$ and $r$ of 0.3 was found to give best agreement with pressure transducer measurements. Changes in the gas composition are expected to play a significant role in affecting the quenching parameters. In particular the presence of water vapour, $\mathrm{H}_{2} \mathrm{O}$, and of carbon dioxide, $\mathrm{CO}_{2}$, has been shown to have a significant impact on quench rates [25]. As in the case of the measurements in the motored engine, the pressure values derived from LITGS data were within $\pm 10 \%$ of the values obtained from the pressure transducer, but only for pressures above 1 bar and up to the point where the fuel mixture was consumed. The pressure transducer is likely to have an absolute accuracy of around 1\% and so the accuracy of the pressure values derived from the LITGS measurements, as defined above, is estimated by comparison with the transducer value.

These results, as those in the cell, indicate that LITGS does provide a viable method for time- and space-resolved measurement of pressure in both static and dynamic situations. The model-fitting method does, however, require significant processing time. Typically the cycle of model fitting to derive pressure values for 1000 data points takes approximately $1 \mathrm{~h}$ on a laptop computer. Strategies for improving the speed of calculation to obtain pressure values in approximately "real time" are underway in our laboratory.

\subsection{Measurements of pressure in a flame}

The same laser system used for the cell-based measurements in NO: $\mathrm{N}_{2}$ mixtures was used to generate LITGS signals in

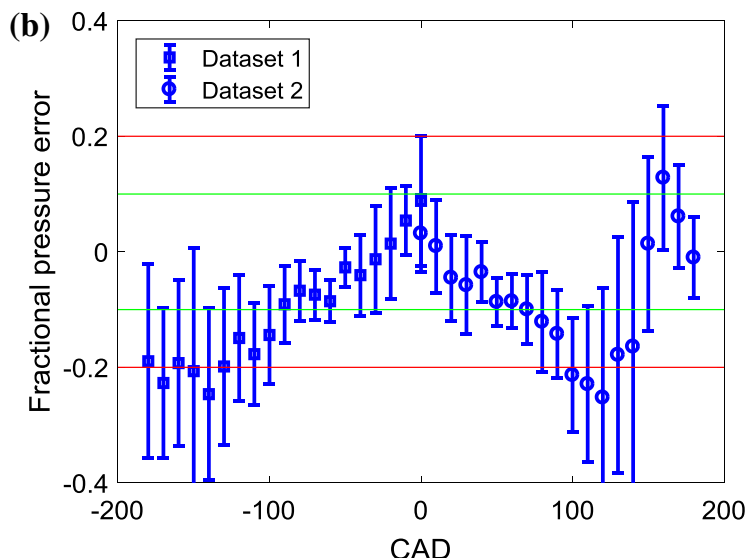

shown (red solid line). The change in the fractional error (relative to the pressure transducer value) is shown in $\mathbf{b}$ for both datasets. The error bars represent the standard deviation of the data points (see text for discussion)

combustion-generated NO. A stoichiometric premixed $\mathrm{CH}_{4} /$ $\mathrm{O}_{2} / \mathrm{N}_{2}$ flame with $\mathrm{O}_{2} / \mathrm{N}_{2}$ ratio $25 / 75$ was established on a small burner to create a high temperature to produce a sufficient concentration of NO to provide LITGS signals with adequate $\mathrm{S} / \mathrm{N}$ ratio. The burner was enclosed in an in-houseconstructed chamber which allowed measurements to be made over the range of 1-2.5 bar.

Although good fits to the experimental data could be obtained, and accurate and precise temperatures were derived it was not possible to derive accurate pressure values [26]. The main difficulty lies in finding an accurate value for the gas viscosity in the flame which is strongly dependent on temperature and composition. The NIST database used throughout this work does not provide reliable values for viscosity at flame temperatures and the viscosity cannot be calculated for mixtures containing high concentrations of water vapour [19]. Furthermore the NIST data acknowledges that values for viscosity of $\mathrm{N}_{2}$ are not accurate above $1800 \mathrm{~K}$ and so large errors are expected at the flame temperatures exceeding $2000 \mathrm{~K}$ encountered in the present work. Making the coarse approximation that the gas properties are dominated by $\mathrm{N}_{2}$, a pressure derived from the best-fit value for the Reynolds number gave a value of 1.7 bar in comparison to a value of 2.5 bar registered by the pressure gauge. These results underline the present limitations of LITGS for measurement of pressure in low pressure and high temperature flames.

\subsection{Sensitivity summary}

The sensitivity (i.e. accuracy) of the derived pressure to each of the fitting parameters will now be summarised.

Parameter $\tau_{\mathrm{g}}$ is measured from the oscillation frequency of the LIGS signal. The "time offset", $\tau_{\mathrm{o}}$, is also measured, 
from the time difference between the signal and pump pulse. Small errors in either parameters would lead to substantial pressure error, but since they are initially determined accurately, the fitting routine is always able to deliver a reliable optimized value.

The derived pressure is linearly dependent on the Reynolds number, $R e$.

The quenching parameters, $Q_{1}, Q_{2}$ and $r$, are more challenging because they are not orthogonal and all contribute to the signal shape. However, we note that the lifetime of the decaying tail of the signal is the best indicator of pressure. The "slow" decay rate, $Q_{2}$, is most dominant here, but the value of the branching ratio, $r$, is also important since it governs the quantity of energy delivered in the slow release rate. The "fast" decay rate, $Q_{1}$, by contrast, is responsible for the initial build-up of the signal. We, therefore, minimise the sensitivity to $Q_{1}$ by fitting only to the tail of the signal in the fourth and final step of the algorithm where all parameters can vary. Of all the quenching parameters, the derived pressure remains most sensitive to $Q_{2}$ and $r$. In step three of the fitting algorithm, a calibration methodology is employed to "fix" them across the dataset. Further investigation revealed that changing the time constant associated with $Q_{2}$ by $10 \mathrm{~ns}$ typically induced a pressure change of $4 \%$.

\section{Conclusions}

A viable method for analysing LITGS signals to extract pressure values has been developed using a fitting procedure for simulated signals based on previous theoretical models with calibration measurements and reasonable physical assumptions to quantify the temperature- and pressure-dependent fitting parameters. The use of calibration measurements to optimise the fitting parameters, improved values of gas dynamic parameters by the use of REFPROP data and judicious weighting of the fitting procedure have provided significant improvement in the accuracy and precision of the pressure determination over previous work. This work has demonstrated that this model-fitting method, MFM, provides significant improvement in the accuracy and precision of pressure measurements using LITGS over previous results obtained in the range 10-40 bar. In particular, in cell-based studies, reasonably accurate and precise measurements have been extended down to lower pressures in the range 1-5 bar, at ambient temperatures. Based on averages over 50-100 single shots an accuracy of $1-3 \%$ was obtained. The singleshot precision in this range was 4-7\%.

The method has been applied to barometry in an optically accessible engine under both motored and fired conditions. In the motored case, the accuracy of the measurements varied from \pm 0.1 to 0.4 bar over the range of $0.5-6.5$ bar with single-shot precisions in the range of 5-20\% over the cycle.
The measurements under firing conditions were limited to a range of CAD up to the point at which the toluene absorber was consumed by combustion. Nonetheless, the measurements in this case showed similar single-shot precisions and only a small decrease in accuracy as a result of uncertainty in the gas composition arising from exhaust gas residuals.

The application of the method to flames at relatively low pressures, $1-2.5 \mathrm{bar}$, and high temperatures $\sim 2300 \mathrm{~K}$, illustrated the limitation of the technique. Rapid diffusion at the elevated temperatures results in much shorter signal duration and consequently reduced precision in the measurement of the decay times. Furthermore, a high degree of uncertainty in the relevant gas kinetic and dynamic parameters in flames results in significant experimental error in deriving the pressure under conditions of high temperature and (relatively) low pressure.

In conclusion, this work has demonstrated that LITGS provides a viable method for space- and time-resolved measurement of pressure in both static and dynamic conditions. Accurate and precise measurements have been shown to be possible especially under conditions of ambient temperatures and pressures in excess of 1 bar. These properties should allow application of the method in a range of situations where remote and non-invasive measurement of local pressure is required.

Acknowledgements This work was part funded by the Engineering and Physical Sciences Council (UK) under Grant number EP/K02924X/1.

Open Access This article is distributed under the terms of the Creative Commons Attribution 4.0 International License (http://creativeco mmons.org/licenses/by/4.0/), which permits unrestricted use, distribution, and reproduction in any medium, provided you give appropriate credit to the original author(s) and the source, provide a link to the Creative Commons license, and indicate if changes were made.

\section{References}

1. A.Y. Chang, B.E. Battles, R.K. Hanson, Simultaneous measurements of velocity, temperature and pressure using rapid $\mathrm{CW}$ wavelength-modulation laser-induced fluorescence of $\mathrm{OH}$. Opt. Lett. 15, 706-708 (1990). https://doi.org/10.1364/OL.15.000706

2. A.Y. Chang, M.D. DiRosa, D.F. Davidson, R.K. Hanson, Rapidtuning $\mathrm{CW}$ laser technique for measurements of gas velocity, temperature, pressure, density and mass flux using NO. Appl. Opt. 30, 3011-3022 (1991). https://doi.org/10.1364/AO.30.003011

3. L.C. Philippe, R.K. Hanson, Laser diode wavelength-modulation spectroscopy for simultaneous measurement of temperature, pressure and velocity in shock-heated oxygen flows. Appl. Opt. 32, 6090-6103 (1993). https://doi.org/10.1364/AO.32.006090

4. M.A. Woodmansee, R.P. Lucht, J.C. Dutton, Development of high-resolution $\mathrm{N}_{2}$ coherent anti-Stokes Raman scattering for measuring pressure, temperature, and density in high-speed gas flows. Appl. Opt. 39, 6243-6256 (2000) 
5. S.P. Kearney, P.M. Danehy, Pressure measurements using hybrid femtosecond picosecond rotational coherent anti-Stokes Raman scattering. Opt. Lett. 40, 4082 (2015)

6. H. Latzel, A. Dreizler, T. Dreier, J. Heinze, M. Dillmann, W. Stricker, G.M. Lloyd, P. Ewart, Thermal grating and broadband degenerate four wave mixing spectroscopy of $\mathrm{OH}$ in high pressure flames. Appl. Phys. B 67, 667 (1998)

7. R. Stevens, P. Ewart, Optical diagnostics of pressure and temperature using laser induced thermal gratings, in Optical and Laser Diagnostics, Institute of Physics Conference Series No. 177, ed. by C. Arcoumanis, K.T.V. Grattan (IoP Publishing, Bristol, 2002)

8. R. Stevens, P. Ewart, Single-shot measurement of temperature and pressure using laser-induced thermal gratings with a long probe pulse. Appl. Phys. B 78, 111 (2004)

9. R. Stevens, P. Ewart, Simultaneous single-shot measurement of temperature and pressure along a one-dimensional line by use of laser-induced thermal grating spectroscopy. Opt. Lett. 31(8), 1055-1057 (2006)

10. E.B. Cummings, Laser-induced thermal acoustics: simple accurate gas measurements. Opt. Lett. 19, 1361 (1994)

11. W. Hubschmid, B. Hemmerling, A. Stampanoni-Panariello, Rayleigh and Brillouin modes in electrostrictive gratings. J. Opt. Soc. Am. B 12, 1850-1854 (1995)

12. J. Kiefer, and P. Ewart Laser diagnostics and minor species detection in combustion using resonant four-wave mixing:. Prog. Energy Combust. Sci. 37(5), 525-564 (2011)

13. R.C. Hart, G.C. Herring, R.J. Balla, Pressure measurement in supersonic air flow by differential absorptive laser-induced thermal acoustics. Opt. Lett. 32, 1689 (2007)

14. P.H. Paul, R.L. Farrow, P.M. Danehy, Gas-phase thermal-grating contributions to four-wave mixing. J. Opt. Soc. Am. B 12, 384 $392(1995)$

15. A. Stampanoni-Panariello, D.N. Kozlov, P.P. Radi, B. Hemmerling, Gas phase diagnostics by laser-induced gratings: I. Theory. Appl. Phys. B 81, 101-111 (2005)

16. B. Hemmerling, D.N. Kozlov, Collisional relaxation of singlet $\mathrm{O}_{2}\left(b^{1} \Sigma_{g}{ }^{+}\right)$in neat gas investigated by laser-induced grating technique. Chem. Phys. 291, 213 (2003)

17. W. Hubschmid, B. Hemmerling, Relaxation processes in singlet $\mathrm{O}_{2}$ analyzed by laser-induced gratings. Chem. Phys. 259, 109 (2000)
18. F. Förster, C. Crua, M. Davy, P. Ewart, Temperature measurements under diesel engine conditions using laser induced grating spectroscopy. Combust. Flame 199, 249 (2019)

19. E.W. Lemmon, M.L. Huber, M.O. McLinden, NIST Standard Reference Database 23, Reference Fluid Thermodynamic and Transport Properties-REFPROP, Version 9.1, National Institute of Standards and Technology (Standard Reference Data Program, Gaithersburg, 2013)

20. B.A.O. Williams, M. Edwards, C.R. Stone, J. Williams, P. Ewart, High precision in-cylinder gas thermometry using Laser Induced Gratings: quantitative measurement of evaporative cooling with gasoline/alcohol blends in a GDI optical engine. Combust. Flame 161, 270-279 (2014)

21. P. Ewart, A. Modeless, Variable bandwidth, tunable laser. Opt. Commun. 55, 124 (1985)

22. M.C. Weikl, F. Beyrau, A. Leipertz, Simultaneous temperature and exhaust-gas recirculation-measurements in a homogeneous charge-compression ignition engine by use of pure rotational coherent anti-Stokes Raman spectroscopy. Appl. Opt. 45,, 36463651 (2006). https://doi.org/10.1364/AO.45.003646

23. C. Willman, P. Ewart, Multipoint temperature measurements in gas flows using $1 \mathrm{D}$ laser induced grating scattering. Exp. Fluids 57, 1 (2016)

24. B. Scott, C. Willman, B. Williams, P. Ewart, R. Stone, D. Richardson, In-cylinder temperature measurements using laser induced grating spectroscopy and two-colour PLIF. SAE Int. J. Engines 10(4), 2191-2201 (2017). doi.https://doi. org/10.4271/2017-24-0045

25. W. Hubschmid, Molecular relaxations in mixtures of $\mathrm{O}_{2}$ with $\mathrm{CO}_{2}$ observed on laser-induced gratings. Appl. Phys. B 94, 345-353 (2009)

26. A. Luers, A.L. Salhlberg, S. Hochgreb, P. Ewart, Flame thermometry using laser-induced-grating spectroscopy of nitric oxide. Appl. Phys. B 124:43 (2018). https://doi.org/10.1007/s0034 0-018-6912-2

Publisher's Note Springer Nature remains neutral with regard to jurisdictional claims in published maps and institutional affiliations. 